Draft Version June 16, 2021

Preprint typeset using $\mathrm{LAT}_{\mathrm{E}} \mathrm{X}$ style emulateapj v. 5/2/11

\title{
UNRAVELLING THE MYSTERIES OF THE LEO RING: AN ABSORPTION LINE STUDY OF AN UNUSUAL GAS CLOUD*
}

\author{
J. L. Rosenberg ${ }^{1}$ And Karl Haislmaier ${ }^{1,2}$ \\ School of Physics, Astronomy, and Computational Science, George Mason University, Fairfax, VA 22030, USA and \\ Department of Astronomy, University of Massachusetts, Amherst, MA 01003, USA
}

M. L. Giroux

Department of Physics and Astronomy, East Tennessee State University, Johnson City, TN, 37614, USA

B. A. KeEney

Center for Astrophysics and Space Astronomy, Department of Astrophysical and Planetary Sciences, University of Colorado, 389 UCB, Boulder, CO 80309, USA

AND

S. E. SCHNEIDER

Department of Astronomy, University of Massachusetts, Amherst, MA 01003, USA

Draft version June 16, 2021

\begin{abstract}
Since the 1980's discovery of the large $\left(2 \times 10^{9} \mathrm{M}_{\odot}\right)$ intergalactic cloud known as the Leo Ring, this object has been the center of a lively debate about its origin. Determining the origin of this object is still important as we develop a deeper understanding of the accretion and feedback processes that shape galaxy evolution. We present HST/COS observations of three sightlines near the Ring, two of which penetrate the high column density neutral hydrogen gas visible in $21 \mathrm{~cm}$ observations of the object. These observations provide the first direct measurement of the metallicity of the gas in the Ring, an important clue to its origins. Our best estimate of the metallicity of the ring is $\sim 10 \% \mathrm{Z}_{\odot}$, higher than expected for primordial gas but lower than expected from an interaction. We discuss possible modifications to the interaction and primordial gas scenarios that would be consistent with this metallicity measurement.

Subject headings: galaxies: abundances — galaxies: ISM
\end{abstract}

\section{INTRODUCTION}

Over twenty-five years ago a giant ring of neutral hydrogen gas (diameter of $\sim 200 \mathrm{kpc}$ and $\mathrm{H}$ I mass of 2 $\left.\times 10^{9} \mathrm{M}_{\odot}\right)$ was found in the Leo region (Schneider et al. 1983; Schneider 1985; Schneider et al. 1986; Schneider 1989; Schneider et al. 1989). At the time, the ring was subject to speculation about its origins - whether it is the remnant of a tidal interaction or is primordial gas left over from the formation of the group of galaxies in which it sits.

The $21 \mathrm{~cm}$ observations provide gas velocities so the original observations of the ring indicated a velocity gradient around the ring that implied that the ring is rotating around a kinematic center that is $15 \pm 7 \mathrm{~km} \mathrm{~s}^{-1}$ from the luminosity weighted center of M105. The highest velocities of the gas are $\sim 1100 \mathrm{~km} \mathrm{~s}^{-1}$ in the southwest and the lowest velocities of $\sim 750 \mathrm{~km} \mathrm{~s}^{-1}$ in the northeast of the ring (Schneider 1985; Havnes et al. 2011, Osterloo et al. private communication). The gas velocities translate to an orbital period of $4 \times 10^{9}$ years.

Since that time, the ring has been the subject of ad-

jrosenb4@gmu.edu

* Based on observations obtained with the NASA/ESA Hubble Space Telescope, which is operated by the Association of Universities for Research in Astronomy, Inc., under NASA contract NAS5-26555. These observations are associated with program GO12198.01-A. ditional studies aimed at providing clues to its origins. Multiwavelength observations of the ring have shown strong limits on the stellar emission within the ring. Early V and K-band observations set the surface brightness limits, at the 3 highest density peaks identified in Skrutskie et al. (1984), as 28.0 and $22.8 \mathrm{mag} \mathrm{arcsec}^{-2}$ respectively. This lack of significant stellar emission in the ring indicates that if it is a remnant of the disruption of a galaxy, the region of the galaxy that was disrupted had to be very gas-rich with a low optical surface brightness.

More recent $r$-band (from the KPNO 4-meter) and FUV and NUV (from GALEX) observations identify faint overdensities of sources in the vicinity of the highest H I column density region. Population synthesis modeling of these optical/UV overdensities are claimed to indicate metallicities of $\mathrm{Z}_{\odot} / 50$ (Thilker et al. 2009). Such low metallicities are consistent with a primordial origin for the ring but not with a tidal origin. However, in addition to the measurement of metallicity from population synthesis, there is a tentative detection of dust emission at $8 \mu \mathrm{m}$ in the densest region of the ring (Bot et al. 2009). The detection, if confirmed, implies a gas-to-dust ratio between $1 / 20$ and a few times solar. This level of dust is inconsistent with the low metallcity derived by Thilker et al. (2009) and is much more consistent with a tidal origin for the gas.

In addition to NGC 3384, M105, M96, and NGC 3351, 
the bright galaxies in the vicinity of the ring, there are 6 lower luminosity galaxies that have been identified (Stierwalt et al. 2009). For two (AGC 201970 and AGC 202027) of these 6 galaxies, the optical velocities place them at a redshift consistent with that of the ring. A third galaxy (AGC 205505) has an optical velocity just above that of the ring but still within the group. The remaining 3 galaxies do not have optical redshifts so their velocities, relative to that of the ring, are not known. Stierwalt et al. (2009) suggest that these galaxies may have formed in situ as tidal dwarf galaxies. These galaxies do not help resolve the question of the origin of the gas since their metallicity is unknown. The existence of tidal dwarf galaxies associated with the Ring is consistent with both scenarios of the feature's origin and would aid in our understanding of the feature if their metallicity was known.

While the studies of Thilker et al. (2009) and Sil'chenko et al. (2003) have argued that the gas is primordial, the connection between the gas in the Ring and the galaxies NGC 3384 and M 96 (as observed in the $21 \mathrm{~cm}$ map, see Figure 10 is often used as evidence of a tidal origin. Rood and Williams (1985) propose a headon or Spitzer-Baade collision between NGC 3384 and M105 while Michel-Dansac et al. (2010) also argue for a head-on collision but consider NGC 3384 and M96 to be the more likely candidates. Alternatively, Bot et al. (2009) argue that their mid-infrared detection of dust associated with the densest part of the ring is consistent with the gas left over from the stripping of a low surface brightness galaxy as has been modeled by Bekki et al. (2005).

The global kinematics have provided additional constraints on the origin of the Leo Ring. Observations of the Ring show a smooth gradient of velocity from $\sim 1100$ $\mathrm{km} \mathrm{s} s^{-1}$ in the southwest down to $\sim 650 \mathrm{~km} \mathrm{~s} s^{-1}$ in the northeast. Schneider (1985) have modeled the velocities as a rotating ring of gas with a kinematic center that is $15 \pm 7 \mathrm{kpc}$ from the luminosity weighted centroid of the M105/NGC 3384 system and an orbital period of $4 \times 10^{9}$ years, longer than the crossing time of the system. On the scale of the individual clouds, the gas exhibits a high velocity disperion that probably arises from multiple blended clumps along the line of sight. In addition, there is evidence for a more diffuse medium between the clumps (Schneider et al. 1986).

Evidence has been accumulating on both sides of the debate about the origins of this unusual object without resolving the conflict. In that context, we present Cosmic Origins Spectrograph (COS) observations of three QSOs that lie behind the ring. We use these observations to study the metallicity and ionization properties of the ring. $\S 2$ discusses the COS observations of these 3 sightlines, $\S 3$ describes the data reduction that was done, $\S 4$ presents ionization modeling of the sightlines, and $\S 5$ provides a discussion of the results. Throughout the paper we use a distance of $10.4 \mathrm{Mpc}$ for the ring, the average distance to the five large galaxies in the region determined from well-established distance indicators (Harris et al. 2007).

\section{OBSERVATIONS}

We have obtained HST/COS spectra at the positions of three QSOs that lie behind the Leo Ring. Table 1 lists the QSOs and their positions are shown overlaid on the Westerbork (Osterloo et al. priv. comm.) and Arecibo (Haynes et al. 2011) H I $21 \mathrm{~cm}$ maps of the Leo Ring in Figure 1] We observe SDSS J104816.25+120734.7 (Q104816 hereafter) and J104709.83+130454.6 (Q104710 hereafter) with the G130M grating and observe SDSS J104843.49+130605.9 (Q104843 hereafter) with both the G130M and G160M gratings. The details of the observations including the exposure times in each grating are given in Table 1].

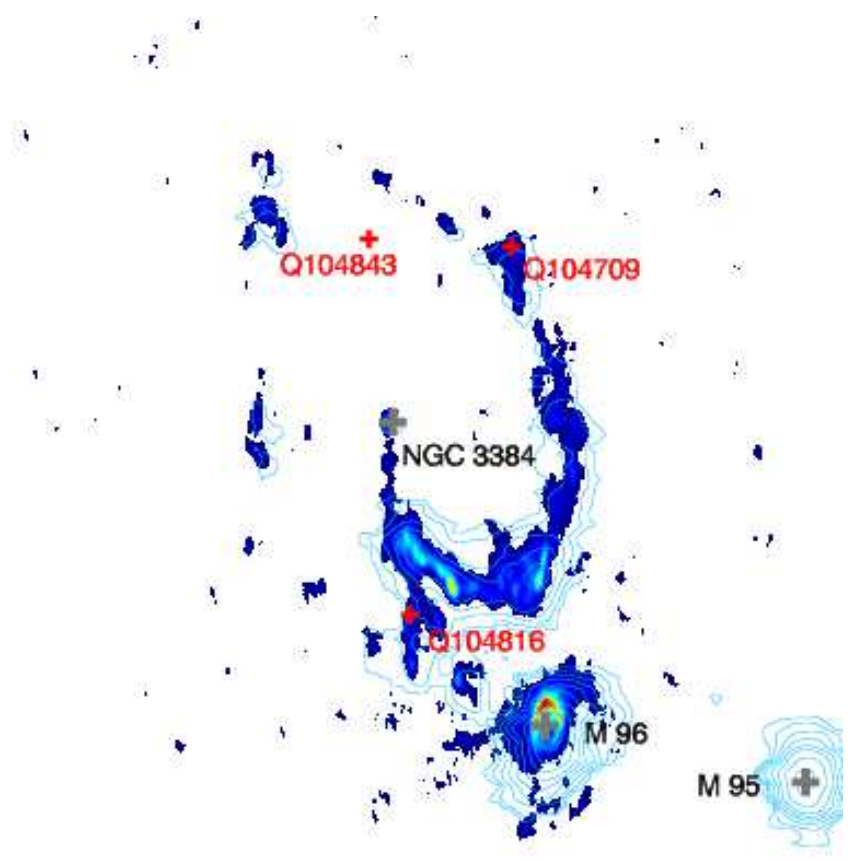

Figure 1. H I $21 \mathrm{~cm}$ observations of the Leo Ring. The color scale shows the H I column density from the Westerbork map (Osterloo et al. priv. comm.). Contours are from the Arecibo telescope as part of the ALFALFA survey (Stierwalt et al. 2009; Havnes et al. 2011). The contour levels are at 5, 15, 30, 50, 120, 300, and 400 $\times 10^{20} \mathrm{~cm}^{-2}$. The positions of M96 and M95 are labeled in the map. Note that M95 is outside of the area covered by the Westerbork map. The red circles indicate the positions of the three QSOs behind the Leo Ring.

Q104816 probes the dense gas $\left(\log \mathrm{N}_{H I} \sim 19.5 \mathrm{~cm}^{-2}\right.$ as measured in the Westerbork emission map) at the Southern edge of the ring a bit south of the densest region region where young stars have been detected by GALEX (Thilker et al. 2009) and dust may have been detected by Spitzer (Bot et al. 2009). Q104709 probes a slightly lower column density $\left(\log \mathrm{N}_{H I} \sim 19.1 \mathrm{~cm}^{-2}\right)$ region than Q104816 in the Northwest region of the Ring. Q104843 is not behind the detected $\mathrm{H}$ I emission contours, but it is just below the ring on the Northern edge (see Figure 1).

\subsection{Absorption Line Measurements using HST/COS Spectra}

The CORRTAG files were extracted from the archive and one-dimensional spectra were extracted using CALCOS V2.19.4. Previous versions of CALCOS calculate errors for the flux values using the Gaussian approximation for Poisson errors $\left(\sigma_{i} \approx \sqrt{N}\right.$, where $N_{i}$ is the gross counts at pixel $i$ ). This assumption systematically underpredicts 
the errors for small values of $N_{i}$, and since our targets are quite faint $(\gtrsim 90 \%$ of the pixels in every exposure have $\left.N_{i} \leq 5\right)$ it is important that we treat the errors in this regime appropriately. Starting with version 2.19.4, CALCOS uses the formalism of Gehrels (1986), which was specifically designed to be valid for all values of $N_{i} \geq$ 0 , to determine the Poisson errors for the flux values.

After the extraction, cross correlation and coaddition of the one-dimensional spectra were then performed with custom routines developed by the COS GTO team specifically for COS FUV datd as described in Danforth et al. (2010) and Keeney et al. (2012). After the creation of the one-dimensional spectra, continua are fit to the coadded spectra using a semi-automated routine described in Keeney et al. (2013). These continuum fits are used to normalize the data for easier absorption line fitting.

Measurements of the O I, N I, and the Ly $\alpha$ line require additional processing of the spectra to mitigate the effects of terrestrial airglow. The modest recession velocity of the Leo Ring $\left(c z \approx 800-900 \mathrm{~km} \mathrm{~s}^{-1}\right)$ toward our QSO sight lines causes the O I $\lambda 1302.2 \AA$, N I $\lambda 1199.5,1200.2$, and $1200.7 \AA$ and $\operatorname{Ly} \alpha \lambda 1215 \AA$ absorption features to fall in regions of the spectrum that suffer from terrestrial airglow emission. To identify the times when the terrestrial airglow was significant we used the CORRTAG files returned from the archive. Orbital night was defined to be times when the zenith distance from the sun was $>95^{\circ}$. When the separation from the sun was larger than this value, the contribution from O I $\lambda 1304.9 \AA$ airglow emission was, emipirically, found to be minimized. The CORRTAG files for each exposure were then split into separate files for orbital day and orbital night and one-dimensional spectra were extracted using CALCOS v2.19.4. The resulting coadded spectra for each sight line incorporate the full (orbital day + night) exposure time for the vast majority of wavelengths and night-only data in regions affected by terrestrial airglow emission. This procedure does not completely remove $\mathrm{H}$ I Ly $\alpha$ airglow emission from the coadded spectrum but it virtually eliminates the $\mathrm{O}$ I and N I airglow emission, at the expense of larger error values in these regions. The spectra created from these split data were then used to estimate limits on the H I Ly $\alpha$ absorption for the three sightlines. We were also able to make a measurement of $\mathrm{N}(\mathrm{N}$ I) along the Q104816 sightline, but neither a measurement nor a limit on $\mathrm{N}(\mathrm{O}$ I) was possible. We were unable to make measurements or obtain meaningful limits on $\mathrm{N}(\mathrm{N}$ I) or $\mathrm{N}(\mathrm{O}$ I) for the other sightlines.

Voigt profile fits to the absorption lines of interest were performed using custom IDL routines that convolve the idealized Voigt profile with the COS on-orbit line spread function (Kriss 2011) to properly account for instrumental resolution effects. All atomic data needed to generate Voigt profiles with a given Doppler parameter and column density were taken from Morton (2003). The results of the line fitting are presented in Tables 2, 3, and 4 for the sightlines Q104816, Q104710, and Q104843 respectively. From the fit to each of the lines, column density, recession velocity, and doppler parameter measurements along with their uncertainties are returned. We use single component fits to the data for these lines.

2 IDL routines for coadding COS data are available at http://casa.colorado.edu/\$\sim\$danforth/science/cos/costools.hthin units of $10^{-23} \mathrm{erg} \mathrm{cm}^{-2} \mathrm{~s}^{-1} \mathrm{~Hz}^{-1} \mathrm{ster}^{-1}$, and con-
Fit parameters SL, b, log N, velocity and the associated errors are only listed once for each atomic species since all of the lines of that species were fit simultaneously. Details of the spectral fitting and evaluation of the uncertainties can be found in Keeney et al. (2013). Tables 2, 3, and 4]also include a measurement of the significance level, $S L$, of each line following the formalism outlined in Keeney et al. (2012).

\section{IONIZATION MODELING OF SIGHTLINES}

We use the measurements of metal-line absorption along the three QSO sightlines to constrain the amount of ionized gas and the metallicity associated with the Leo Ring gas. We adopt solar values for metal abundances (on a logarithmic scale) from Asplund et al. (2009) of $[\mathrm{H}]=12.00,[\mathrm{C}]=8.43,[\mathrm{~N}]=7.83,[\mathrm{O}]=8.69$, and $[\mathrm{Si}]=7.51$.

Because we only observe select ionization states for the elements that we are examining, we must make ionization corrections to calculate the total column density of a given element from the measured column density of a single ionization state. As an example, the ionization corrections for Si are defined as:

$$
[\mathrm{Si} / \mathrm{H}]=[\mathrm{S} \mathrm{II} / \mathrm{H} \mathrm{I}]+(\text { correction })
$$

where logarithmic quantities are implied. Such corrections are uncertain even if the gas is primarily photoionized because they depend on the gas density and the properties of the ionizing radiation field. We also neglect the possibility of depletion.

In order to estimate ionization corrections we have constructed a grid of photoionization models associated with each sightline using the Cloudy modelling code (Ferland et al. 1998, version 10.00). We model the absorption as a single, constant density (one phase), plane parallel slab illuminated by a uniform incident radiation field. We consider two spectral shapes for the incident radiation field based upon the calculations of the metagalactic radiation field of Haardt \& Madau (as implemented within Cloudy based upon personal communication from those authors). In the first model, the incident radiation field includes emission from both QSOs and galaxies. This model assumes the contribution from galaxies to the hydrogen ionizing spectrum is approximately equal to that from QSOs. The incident radiation field in the second model ("QSO-only") assumes a background solely due to QSOs. This model has a higher proportion of high energy photons and may represent a limit in which few ionizing photons escape from galaxies.

The ionization corrections are dependent on the ionization parameter $U=n_{\gamma} / n_{H}$, where $n_{\gamma}=\Phi / c$ is the density of photons above the hydrogen ionization potential, and $n_{H}$ is the hydrogen density. The quantity $\Phi$ is the surface flux of hydrogen ionizing photons gen ionizing photons assumed in the first Haardt \& Madau model (galaxies plus QSOs) is $\Phi_{H M}=3.2 \times$ $10^{4}$ photons $\mathrm{cm}^{-2} \mathrm{sec}^{-1}$. This value depends on the level of the metagalactic radiation field at $z=0$, and is a quantity that remains uncertain. The level of the metagalactic radiation field has been expressed in terms of $\Phi$ as well as in terms of $\Gamma$, the hydrogen photoionization rate in units of $\mathrm{s}^{-1}$, and $J_{-23}$, the mean intensity (photons $\mathrm{cm}^{-2} \mathrm{~s}^{-1}$ ). The surface flux $\Phi_{H M}$ of hydro- 
straints are usually expressed in terms of one of these quantitities. There is a weak spectral dependence on the relation between these values, but approximately, for $\Phi_{H M}=3.2 \times 10^{4}, \Gamma \approx 7 \times 10^{-14}$, and $J_{-23} \approx 3$.

For the cases where we have measurements or limits on higher ionization species such as Si III, Si IV, and C IV, we determine whether the values are consistent with the conditions determined for the lower ionization species. Because these more highly ionized species are often associated with a different phase of the gas we do not apply the results directly but consider whether they provide evidence for a more highly ionized phase.

If we assume $\Phi$, ( or $\Gamma$ or $\left.J_{-23}\right)$ is known, we can translate the ionization parameter $U$ to an inferred density $n_{H}$ and, in combination with an observed H I column density, can translate this into a line-of-sight extent of the gas. Assuming that the line-of-sight extent of the gas is unlikely to greatly exceed the lateral extent, we can constrain the allowed range of $U$. The success of this exercise depends on how well the level of the metagalactic background at $z=0$ is known. Estimates of this level have been made based upon the integration of the quasar luminosity function, the line of sight proximity effect, the flux decrement method, and from limits on $\mathrm{H} \alpha$ emission associated with isolated gas clouds. The results of many of these investigations are summarized in Adams et al. (2011, see their Figure 6). In general, these estimates of the metagalactic background are somewhat lower than the fiducial value that is used in the Cloudy models, $\Phi / \Phi_{H M} \lesssim 0.7$. For a given ionization parameter $U$, the line of sight extent of the gas scales roughly with the inverse of $\Phi$. As a result, we assume that estimates of the line of sight extent based upon $\Phi_{H M}$ represent conservative lower limits on the extent of the gas; thus the assumption that the line of sight extent of our model is $<30 \mathrm{kpc}$ places a lower limit on the range of likely ionization parameters.

\subsection{Absorption Line Results for SDSSJ 104816.25+120734.7}

Q104816 lies behind a radio contour corresponding to $\log N(H I)=19.5$ on the H I $21 \mathrm{~cm}$ Westerbork map (Osterloo et al. priv. comm.). The angular resolution of the $\mathrm{H}$ I emission observations are significantly lower than the HST FUV observations $-1.75^{\prime} \times 0.65^{\prime}$ for the radio map in contrast with the subarcsecond pencilbeam associated with the UV absorption line observations. Despite the resolution difference, we do not measure the Ly $\alpha$ absorption directly from the HST/COS spectra that are shown in Figure 2 along with the H I $21 \mathrm{~cm}$ emission profiles from the ALFALFA survey (Haynes et al. 2011) for this and the other 2 sightlines discussed below. We do not use the Ly $\alpha$ absorption to measure the H I content because it is strongly blended with absorption from Milky Way Ly $\alpha$ making a measurement from these data highly unreliable. However, for the Q104816 sightline, the HST/COS data does indicate that the Westerbork value, $\log \mathrm{N}(\mathrm{H} \mathrm{I})=19.5$ is consistent with the Ly $\alpha$ absorption profile.

Figure [3] shows the Q104816 HST/COS spectra and absorption line fits for the Si II $\lambda 1190,1193,1260,1304$, Si III $\lambda 1206$, Si IV $\lambda 1394,1402$, C II $\lambda 1334$, and N I $\lambda 1199,1200$ lines on which we base our results.

The absorption line measurements, line velocities, sig-

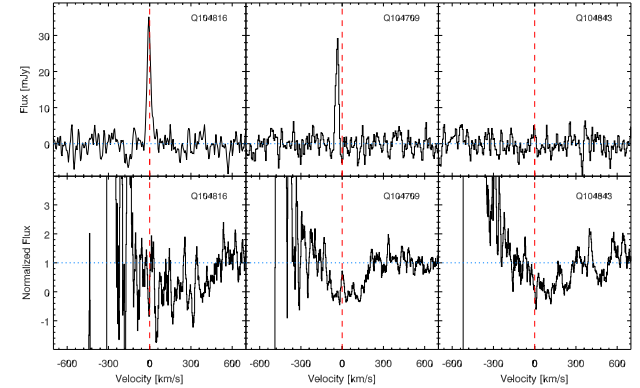

Figure 2. The H I $21 \mathrm{~cm}$ emission lines as measured from the ALFALFA survey (Havnes et al. 2011) for each sightline are shown in the top panel. The corresponding Ly $\alpha$ absorption in the HST/COS spectra, smoothed to $\sim 12 \mathrm{~km} \mathrm{~s}^{-1}$ are shown in the bottom panels. Because of the Ly $\alpha$ airglow emission seen at velocities less than 0 and the Milky Way Ly $\alpha$ absorption, we use the radio H I measurements even though the resolution is much lower for these data.

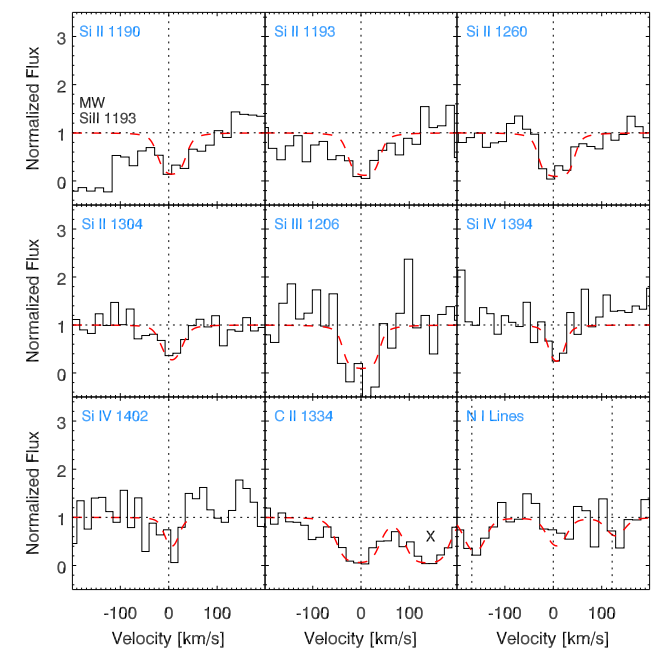

Figure 3. The absorption lines identified and measured in the Q104816 spectrum. The red dashed lines indicate the fits to the lines that were used. The spectra are centered at a velocity of 917 $\mathrm{km} s^{-1}$.

nificance level (SL) of the line fits, and the integrated EW values are given in Table 2. We note that the weighted average of the line velocities is $917 \mathrm{~km} \mathrm{~s}^{-1}, 5$ $\mathrm{km} s^{-1}$ larger than the value measured from the Westerbork H I $21 \mathrm{~cm}$ emission. This difference is within the expected uncertainty in the velocity scale for the HST/COS measurements. Since we do not make use of the line velocities in our analysis, this difference will not have an effect on our results. The resulting column densities along this line of sight are log N(Si II) $=14.4 \pm 0.5 \mathrm{~cm}^{-2}, \log \mathrm{N}(\mathrm{C}$ II $)=14.7 \pm 0.1 \mathrm{~cm}^{-2}$ and $\log \mathrm{N}(\mathrm{N} \mathrm{I})=14.3 \pm 0.4 \mathrm{~cm}^{-2}$.

\subsubsection{A Metallicity Estimate}

We use the measurements of the absorption lines to determine our nominal measurement of the metallicity along this sightline. We use the $\mathrm{H}$ I column density from the Westerbork $21 \mathrm{~cm}$ emission line measurement, log $\mathrm{N}(\mathrm{H} \mathrm{I})=19.5$. This value gives column density ratios with respect to $\mathrm{H}$ I of $[\mathrm{Si} \mathrm{II} / \mathrm{H} \mathrm{I}]=-5.1 \pm 0.5,[\mathrm{C} \mathrm{II} / \mathrm{H} \mathrm{I}]$ 
$=-4.8 \pm 0.1$, and $[\mathrm{N} \mathrm{I} / \mathrm{H} \mathrm{I}]=-5.2 \pm 0.4$. If the fractions in these ionization stages matched the fraction of neutral hydrogen (i.e., no ionization correction is needed), this would imply logarithmic abundances with respect to solar of $-0.6 \pm 0.5,-1.2 \pm 0.1$, and $-1.0 \pm 0.4$ for silicon, carbon, and nitrogen, respectively.

Figure 4 shows the results of a set of photoionization models designed to determine the ionization corrections required to obtain elemental abundances $[\mathrm{Si} / \mathrm{H}],[\mathrm{N} / \mathrm{H}]$, and $[C / H]$. When the ionization corrections, as plotted in Figure 4 (and Figure [6), are negative as in the cases of silicon and carbon, the metallicity corrected for ionization will be lower than the measured ratios. Alternatively, when the ionization correction is positive, as it is for nitrogen, the metallicity will be higher than the measured ratio. Requiring the line of sight extent of the gas to be less than $30 \mathrm{kpc}$ constrains the gas to have $\log U<-3.1$ for $\Phi_{H M}=3.2 \times 10^{4}$ phot $\mathrm{cm}^{-3} \mathrm{sec}^{-1}$ and $\log N(\mathrm{H} \mathrm{I})=19.5$. The largest ionization corrections required are -0.4 , for $[\mathrm{S} \mathrm{II} / \mathrm{H} \mathrm{I}]$ and $[\mathrm{C} \mathrm{II} / \mathrm{H} \mathrm{I}]$ and 0.2 for $[\mathrm{N} \mathrm{I} / \mathrm{H} \mathrm{I}]$ so the metallicities corrected for ionization are $[\mathrm{Si} / \mathrm{H}]=-5.5_{-0.4}^{+0.9},[\mathrm{C} / \mathrm{H}]=-5.2_{-0.1}^{+0.5}$, and $[N / H]=-5.0_{-0.6}^{+0.4}$. The errorbars on these measurements conservatively include the ionization corrections since they are uncertain. Expressing these values in terms of solar abundances, these values correspond to $[\mathrm{Si} / \mathrm{H}]_{\odot}=-1.0_{-0.4}^{+0.9},[\mathrm{C} / \mathrm{H}]_{\odot}=-1.6_{-0.1}^{+0.5}$, and $[N / H]_{\odot}=-0.8_{-0.6}^{+0.4}$.

For comparison, Schneider et al. (1986) measured the properties of gas clumps identified in the ring. They inferred densities for the clumps of $-1.8<\log n_{H I}<-0.6$ $\mathrm{cm}^{-3}$. However, since these clumps represent $\lesssim 0.5$ of the integrated flux they also infer a more diffuse medium with $\log n_{H I} \lesssim-3 \mathrm{~cm}^{-3}$. Our values for $U$ and $\Phi$ correspond to $\log n(H)>-2.9 \mathrm{~cm}^{-3}$ which is approximately $\log n_{H I}>-4.3 \mathrm{~cm}^{-3}$, consistent with either a dense phase or a more diffuse phase. Our ionization corrections assume that the gas is associated with the more diffuse phase, but the denser phase would imply smaller ionization corrections and thus higher abundances of carbon and silicon (and marginally lower abundances of nitrogen).

The measured values for the ratios, $[\mathrm{Si} \mathrm{III} / \mathrm{Si} \mathrm{II}]=$ $1.6 \pm 1.4$, and $[\mathrm{Si} \mathrm{IV} / \mathrm{Si} \mathrm{II}]=-0.6 \pm 0.3$, are just barely consistent with the models at $\log n_{H} \approx-3$ for an AGN plus stellar ionizing background.

\subsection{Absorption Line Results for SDSSJ $104709.83+130454.6$}

Q104709 lies behind a radio contour corresponding to $\log N(H I)=19.1$ on the H I $21 \mathrm{~cm}$ Westerbork map (Osterloo et al. priv.comm.). A nominal fit to the Lyo absorption indicates a column density of $\log N(H I)=$ $18.8 \pm 0.3$. As with the previous sightline, this region of the spectrum has a very low $\mathrm{S} / \mathrm{N}$ due to the damping wings of Galactic $\operatorname{Ly} \alpha$ and a smaller exposure time because daytime data were eliminated to reduce the $\operatorname{Ly} \alpha$ airglow. Given the difficulties with the absorption line measurement, we adopt the Westerbork measurement of $\log N(H I)$ rather than the measurement from the HST/COS spectrum as we did for Q104816 because it is strongly blended with absorption from Milky Way Ly $\alpha$.

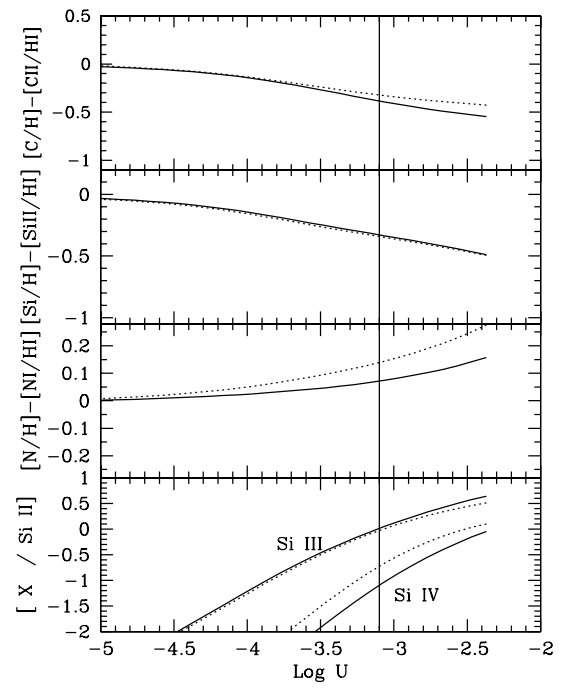

Figure 4. The top three panels represent ionization corrections for $\mathrm{C}, \mathrm{Si}$, and $\mathrm{N}$, respectively (see Equation 1) versus $\log U$ where $U$ is the ionization parameter. The solid curves assume the AGN plus galaxies metagalactic radiation field while the dotted curves assume an AGN-only shape to the radiation field. The bottom panel represent model ratios of Si III/Si II and Si IV/Si II, as labeled, versus $\log U$. The vertical line through all panels represents the maximum allowed value of $\log U$ assuming a maximum line of site extent of $30 \mathrm{kpc}$ and $\Phi_{H M}=3.2 \times 10^{4}$ photons $\mathrm{cm}^{-2} \mathrm{sec}^{-1}$ (see $\S 4$ ). All models assume $\log N(H I)=19.5$, appropriate for the Q104816 sightline.

The spectrum is shown in Figure 2 along with the ALFALFA emission profile at this position.

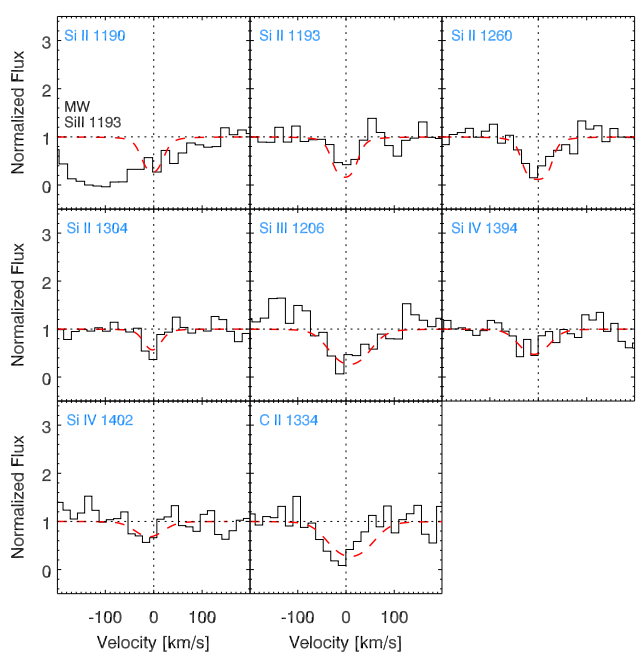

Figure 5. The metal line absorbers identified and measured in the HST/COS spectrum of Q104709. The red dashed lines show the fits to the lines that were used. The velocity is centered at 855 $\mathrm{km} \mathrm{s}{ }^{-1}$.

Figure 5 shows the HST/COS spectrum and absorption line fits for the Si II $\lambda 1190,1193$, and 1260 , Si III $\lambda 1260,1304$, Si IV $\lambda 1394$ and 1402 , and C II $\lambda 1334$ lines on which we base our results.

The absorption line measurements, line velocities, significance level (SL) of the line fits, and the integrated EW values are given in Table 3. We note that the 
weighted average of the line velocities is $857 \mathrm{~km} \mathrm{~s}^{-1}, 38$ $\mathrm{km} s^{-1}$ larger than the value measured from the Westerbork H I $21 \mathrm{~cm}$ emission. This difference is larger than the expected uncertainty in the velocity scale for the HST/COS measurements, $15 \mathrm{~km} \mathrm{~s}^{-1}$, possibly a combination of the velocity scale uncertainty and the low $\mathrm{S} / \mathrm{N}$ of the data.

\subsubsection{A Metallicity Estimate}

We use the measurements of the absorption lines to determine our nominal measurement of the metallicity for Q104709 as we did for Q104816. The resulting column densities along this line of sight are $\log \mathrm{N}(\mathrm{Si}$ II $)=14.0 \pm$ $0.2 \mathrm{~cm}^{-2}$ and $\log \mathrm{N}(\mathrm{C} \mathrm{II})=14.5 \pm 0.1 \mathrm{~cm}^{-2}$. For $\log$ $\mathrm{N}(\mathrm{H} \mathrm{I})=19.1$, the column density ratios with respect to $\mathrm{H} \mathrm{I}$ are $[\mathrm{Si} \mathrm{II} / \mathrm{H} \mathrm{I}]=-5.1 \pm 0.2,[\mathrm{C} \mathrm{II} / \mathrm{H} \mathrm{I}]=-4.6 \pm 0.1$.

Figure 6 shows the photoionization models used to compute the ionization corrections that were applied to obtain elemental abundances $[\mathrm{Si} / \mathrm{H}]$ and $[\mathrm{C} / \mathrm{H}]$ assuming $\log \mathrm{N}(\mathrm{H} \mathrm{I})=19.1$, Requiring the line of sight extent of the gas to be less than $30 \mathrm{kpc}$ constrains the gas to have $\log U<-3.1$ for $\Phi_{H M}=3.2 \times 10^{4}$ phot $\mathrm{cm}^{-3} \mathrm{sec}^{-1}$ and $\log N(\mathrm{H} \mathrm{I})=19.1$.

The largest ionization corrections consistent with our line of sight constraint are of -0.5 and -0.6 for silicon and carbon respectively. This would imply logarithmic abundances with respect to solar of $[\mathrm{Si} / \mathrm{H}]=-1.1_{-0.2}^{+0.7}$ and $[\mathrm{C} / \mathrm{H}]=-1.0_{-0.1}^{+0.7}$ While the Si III absorption line is not highly significant, we accept the estimate of log $\mathrm{N}$ Si III $=13.4 \pm 0.2 \mathrm{~cm}^{-2}$ and associate it with the same phase that contains C II and Si II. In this case, the bottom panel of Figure 6 shows that the ionization parameter would be less than $\log U \approx-3.5$. A small value for $\log U$ would reduce the ionization required and would increase the abundance estimates for silicon and carbon.

While the Si III and Si IV absorption lines are not highly significant, if their relative strengths are taken at face value they provide strong evidence for the presence of a multiphase medium. As the bottom panel of Figure 6] indicates, a very low gas density (high $U$ ) is required for the Si III and Si IV column densities to be comparable in a single phase medium. Alternatively, the relative Si IV column density could be elevated by assuming that it is in a higher temperature phase than that associated with pure photoionization equilibrium. This warm diffuse phase may have a negligible contribution to the $\mathrm{H}$ I column density but may contain the vast majority of the Si IV and possibly Si III column density along the sightline.

The presence of a higher ionization phase along the sightline should not strongly impact our metallicity estimates associated with the low ionization lines. To the extent that the Si III column density is associated with the higher ionization phase rather than the low ionization phase, it may indicate a smaller ionization correction. This smaller ionization correction, when applied to the low ionization phase, would then imply silicon and carbon abundances approaching the high end of our estimated range.

\subsection{SDSSJ $104843.49+130605.9$}

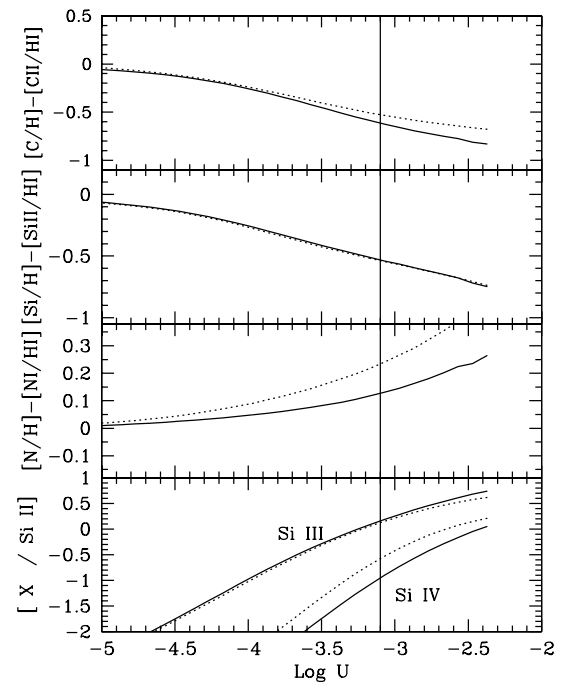

Figure 6. The top three panels represent ionization corrections for $\mathrm{C}, \mathrm{Si}$, and $\mathrm{N}$, respectively (see Equation 2) versus $\log U$ where $U$ is the ionization parameter. The bottom panel shows model ratios of Si III/Si II and Si IV/Si II, as labeled, versus $\log U$. The vertical line through all panels represents the maximum allowed value of $\log U$ assuming a maximum line of site extent of $30 \mathrm{kpc}$ and $\Phi_{H M}=3.2 \times 10^{4}$ photons $\mathrm{cm}^{-2} \mathrm{sec}^{-1}$ (see $\S 4$ ). All models assume $\log N(H I)=19.1$, appropriate for the Q104709 sightline.

No radio emission has been detected at the position of Q104843 implying an upper limit on the average column density of $\log \mathrm{N}(\mathrm{H} \mathrm{I})<18.73 \mathrm{~cm}^{-2}$, although regions within the radio beam could have higher column density if the medium is clumpy. While a definitive measurement of $\mathrm{N}(\mathrm{H} \mathrm{I})$ from the Ly $\alpha$ absorption is not possible, we have compared absorption profiles with a range of $\mathrm{N}(\mathrm{H} \mathrm{I})$ and $\mathrm{b}$-values with the data and have concluded that values of $\mathrm{N}(\mathrm{H} \mathrm{I})$ substantially above (or below) $\log \mathrm{N}(\mathrm{H} \mathrm{I}$ ) $=18.73 \mathrm{~cm}^{-2}$ are not compatible with the HST $/ C O S$ data.

Figure 7 shows the C II $\lambda 1334$, Si III $\lambda 1206$, and C IV $\lambda 1548$ and 1550 lines that are detected along this sightline and the spectral fits upon which we base our tabulated velocities, doppler parameters and column densities along with their formal uncertainties. The summary of our results is given in Table 4. Given the low formal SL's associated with these metal lines, it is difficult to say anything quantitative about the metallicity of the gas along this sightline. The detection of C IV along this sightline provides further indication of a warm diffuse gas component within the Leo Ring and extended beyond the boundaries of the cold neutral component observed in H I.

\subsection{Summary}

We have measured metal-line absorption along three QSO sightlines though the Leo Ring. For the two sightlines that show H I $21 \mathrm{~cm}$ emission, we make use of those detections to compute $\mathrm{N}_{H I}$. This is done despite the beam size mismatch between the two observations because of the uncertainty in the Lyman $\alpha$ absorption measurements (see $\S 3.1$ and $\S 3.2$ for details).

Using the photoionization models discussed in the text we derive the metallicity along the highest column density sightline, Q104816 to be between 2 and 10\% solar. Along the sightline Q104709 we find a nominal metallic- 


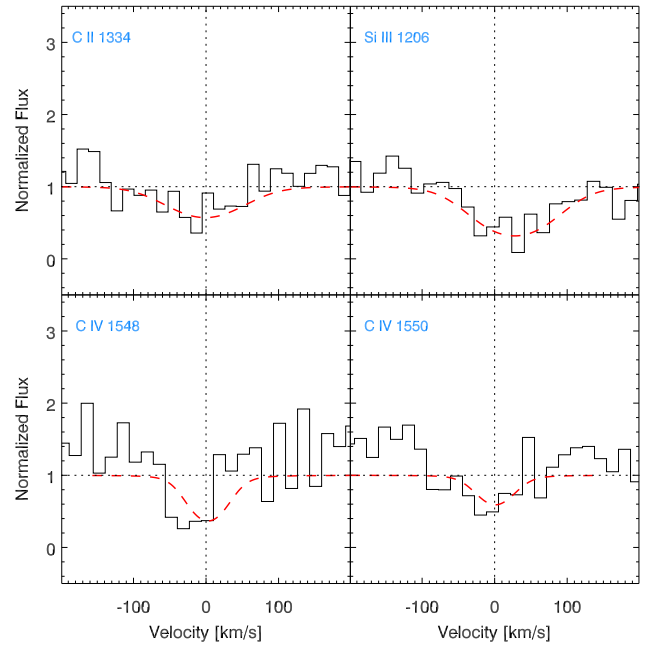

Figure 7. The absorption lines identified and measured in the Q104843 spectrum. The red dashed lines indicate the fits to the lines that were used. The velocity is centered at $844 \mathrm{~km} \mathrm{~s}^{-1}$.

ity of $10-16 \%$ solar and a lower limit of $2 \%$ solar. The third sightline, Q104843, shows metal line absorption, but a quantitative determination of the metallicity can not be made because of the lack of a reliable $\mathrm{H}$ I detection.

The measurements of high ionization species in Q104709 and Q104843 indicate the existance of a higher ionization phase of the gas than what is probed by the lower ionization species. While the higher ionization species in Q104816 are consistent with the lower ions, it may be that there is a higher ionization phase, it is just not significant enough to affect the line ratios.

The uncertainties in our measurements of metallicity are largely from the lack of a Lyman $\alpha$ measurement of the $\mathrm{H}$ I column density of these sightlines and the low signal-to-noise of the data.

\section{DISCUSSION}

This study of the metallicity in the Leo Ring was designed to constrain the origin of the gas. Using the absorption lines identified in the QSO spectra, we find a metallicity of $\sim 10 \% \mathrm{Z}_{\odot}$, marginally higher than the $2 \%$ $\mathrm{Z}_{\odot}$ found by Thilker et al. (2009) but consistent with the measurements of Bot et al. (2009) which, if correct, indicate that there is dust in the ring. We explore the implications of this metallicity with regard to three scenarios for the formation of the Ring: (1) a tidal interaction between M96 and NGC 3384, (2) the disruption of a low surface brightness galaxy by the group, and (3) primordial intergalactic gas.

The discussion of these three scenarios is based on our measurements of the metallicity along these sightlines which are summarized in Table 5. These values have been measured as well as our data will allow, but there is some uncertainty beyond the measured errors. This uncertainty is because the QSO sightlines are not well matched spatially by the $21 \mathrm{~cm}$ beam used to measure the H I column density. The values measured from the Westerbork maps are consistent with the Ly $\alpha$ absorption, to the limits of the QSO data, but the uncertainties are large. If $\mathrm{N}(\mathrm{H} \mathrm{I})$ is larger than we have assumed, the ratios of $[\mathrm{Si} \mathrm{II} / \mathrm{H} \mathrm{I}]$ and $[\mathrm{C} \mathrm{II} / \mathrm{H} \mathrm{I}]$ would be smaller so uncorrected for ionization the abundances would be lower. However, for a given ionization parameter, the ionization correction for a larger H I column density has a smaller absolute value which, in the cases of silicon and carbon, where the ionization corrections are negative, would at least partially offest the decrease in the abundance. However, nitrogen has a positive ionization correction so the errors would tend to add, but the ionization corrections are generally small so the error is dominated by the uncertainty in the $\mathrm{H}$ I column density.

The metallicity in gaseous tidal tails is difficult to determine directly so it is often measured in associated tidal dwarf galaxies. These tidal dwarf galaxies are found to have high metallicities (often near solar or even super-solar; e.g., Torres-Flores et al. 2012, de Mello et al. 2012) for their luminosities - they do not follow the luminosity-metallicity relation (e.g., Duc et al. 2014). This deviation from the relation is probably because material from which they formed is drawn from the inner disk of the galaxy. Michel-Dansac et al. (2010) have modeled the Leo Ring as material removed when M 96 plunged though the center of NGC $3384 \sim 1.2$ Gyr ago. NGC 3384 has a metallicity of 1.1 times solar at $1.25 \mathrm{kpc}$ from the center of the galaxy (Sánchez-Blázquez et al. 2007) and M96 is a very metal-rich galaxy with a metallicity of $\sim 3.2 \mathrm{Z}_{\odot} 3.3 \mathrm{kpc}$ from the center of the galaxy (Oey and Kennicutt 1993). The enrichment found in tidal dwarf galaxies would suggest that if the Leo Ring was formed as a tidal tail resulting from an interaction between these galaxies a metallicity near solar, significantly larger than what we have measured here, would be expected.

However, the Leo Ring, with its circular morphology, looks more like a collisional ring (i.e., the result of a head-on collision between NGC 3384 and M105) than a more generic tidal interaction. Appleton et al. (1996) review the models of collisional rings and show that the rings come from the expansion of the outer regions of the disk and are thus less affected by metallicity mixing than other tidal interactions. In a study of 8 collisional rings Bransford et al. (1998) find metallicities ranging from about quarter solar to solar, closer to but still somewhat higher than found for the Ring. In addition to this metallicity difference, it is also important to note that the Leo Ring is significantly larger than any other collisional ring galaxy known (where the largest are $\lesssim 60$ kpc, Madore et al. 2009) and it lacks the star formation seen in the models and observations of other collisional rings (e.g., Higdon and Wallin 1997; Fogarty et al. 2011; Mapelli and Maver 2012).

As an alternative to looking at the metallicities of tidal dwarf galaxies, which may not be an appropriate model if the material was not drawn from the inner disk of the galaxy, a lower limit on the expected metallicity due to a tidal interaction can be found by extrapolating from the metallicity in the inner region of the disk to the distance of the Ring assuming a metallicity gradient for the galaxy. Since one would expect the gas to arise at smaller radii than the current location of the ring and for some mixing to occur, the projected metallicity of the galaxies at large radii provides a lower limit. Given the central metallicity and gradient for NGC 3384 
(Sánchez-Blázquez et al. 2007), the galaxy would reach $\sim 20 \%$ solar metallicity at $30^{\prime}(90 \mathrm{kpc})$ from the center, the approximate distance of the Ring. The galaxy would not reach $10 \%$ solar metallicity until $>800 \mathrm{kpc}(500 \mathrm{kpc}$ if you use the elevated central metallicity in this calculation). For M 96, assuming the most extreme metallicity gradient $(-0.25)$ seen in the galaxies sampled by Werk et al. (2011) (with the exception of the lower slope for NGC 2146) the metallicity of M 96 would be $\sim 11 \%$ at $90 \mathrm{kpc}$. So assuming that the metallicity is correct for the Ring, it is very difficult to match the metallicity with gas associated from the galaxy without a much steeper metallicity gradient in the outer regions of these galaxies than seen in the inner regions of the disk. In most of the galaxies where metallicity has been studied at large radii flattening of the metallicity gradient is observed (e.g. Werk et al. 2011; Bresolin et al. 2012, 2009; Goddard et al. 2011).

Interactions seem to be the most obvious source for the gas in the Leo Ring - morphologically the gas appears to be connected to an interaction between NGC 3384 and M 96. The tidal scenarios discussed above imply a metallicity that is higher than what has been calculated from the absorption lines that we have measured. Even with the uncertainties in our metallicity measurements, it is difficult to reconcile the low metallicities that we calculate with what we would expect for tidal material surrounding super-solar metallicity galaxies. One possibility in reconciling this model with the data is that there has been significant mixing between tidal material and more primoridal gas. To acheive a metallicity of $10 \%$, the enriched material removed from these systems would have to be a minority component mixed with low metallicity material from the IGM - in the extreme case where gas that is only $20 \%$ solar is removed from the galaxy and mixed with $3 \% \mathrm{Z}_{\odot}$ gas from the IGM, $59 \%$ of the gas would have to come from the IGM to create the $10 \% \mathrm{Z}_{\odot}$ gas in the Ring.

Another scenario that has been proposed for the Ring is the disruption of a low surface brightness galaxy. The galaxy has to have been low luminosity and low surface brightness for there to be no trace of the stars in optical observations of the Ring. Such a system, because of the luminosity-metallicity relationship (e.g., Tremonti et al. 2004), would be expected to be low metallicity. While the inability to detect stars from the galaxy that was disrupted is problematic for this scenario, the metallicity of the gas would be consistent. For comparison, several new metallicity measurements along the Magellanic Stream give a value of $10 \%$ solar, consistent with material removed from the dwarf irregular galaxy, the Small Magellanic Cloud (SMC; Fox et al. 2013). However, the H I mass of the SMC is only $4.2 \times 10^{8} \mathrm{M}_{\odot}$ (Stanimirovic et al. 1999), significantly smaller than the H I mass of the Leo Ring.

The final scenario, initially proposed by Schneider $(1985)$ is that the gas in the Ring is primordial. Sil'chenko et al. (2003) also argued for this scenario after studying the stellar ages and kinematics of the galaxies in the group. They looked at the stellar ages and kinematics of the central regions of NGC 3384, M 96, and M 105 and concluded that all three galaxies have central disks or rings. They argue that those features are aligned, or in the case of the ring in M 105, orthogonal to the line of nodes of the Leo Ring. They use these kinematics and the mean ages of the central stellar populations, which are all $\sim 3 \mathrm{Gyr}$ and close to the $4 \mathrm{Gyr}$ orbit time for the Ring, to argue that the Ring is primordial and is still feeding material into the galaxies. The metallicity result of $2 \%$ solar found by Thilker et al. (2009) would be consistent with this scenario. While our nominal measurement of the metallicity of the Ring is higher than expected given this scenario, we can not rule out a metallicity of a few percent. However, our nominal measurement of metallicity seems to indicate that if the Leo Ring is IGM material, it has been polluted, at least to some degree by material from the surrounding galaxies.

Lehner et al. (2013) and Wotta et al. (2014) studied the metallicity of Lyman-limit systems at $z<1$ and find that they have a bimodal distribution with peaks at $\sim 2.5 \%$ and $\sim 50 \%$ solar metallicity. The higher metallicity material is thought to trace winds, recycled material, and outflows while the more pristine gas is the more primordial material. The gas in the Ring falls between the two branches which may imply a rare mixing of primordial and enriched material. If M 105, the elliptical in the group, was responsible for the enrichment of the gas through outflows that transformed this galaxy to its current gas-poor state, we can look at the metals created in the formation of the galaxy's stellar population to gain insight into the possible enrichment. Using the population synthesis models of Bruzual and Charlot (2003) and assuming that the stellar population of this galaxy was formed in a single burst, the amount of gas returned to the ISM from type II SNe is $2.4-2.5 \times 10^{10} \mathrm{M}_{\odot}$, with the range reflecting differences in possible starting metallicities. Given that the metal yield of SNe averaged over a population of massive stars is expected to be roughly solar in metallicity (Woosley et al. 2002), only $\sim 1.4 \times 10^{8} \mathrm{M}_{\odot}$ of this gas needs to be removed from M105 and mixed with $3 \% \mathrm{Z}_{\odot}$ metallicity material in the IGM to enrich the Ring to the measured level. We use $3 \%$ as the nominal IGM metallicity based on the low metallicity branch of the Lyman-limit cloud distribution measured by Lehner et al. (2013).

Using absorption lines towards three QSOs behind the Leo Ring we have measured the nominal metallicity of the Ring to be $\sim 10 \%$ solar. This metallicity is lower than expected for tidal material stripped in an interaction between M96 and NGC 3384, the primary candidates for such an interaction. Alternatively, it would be consistent with the expected metallicity of a low surface brightness galaxy, but this scenario suffers from the the lack of identification of an optical component to such a system and the need to consider an extremely gas-rich interaction candidate. The nominal metallicity is also higher than expected for primordial material that has not been polluted by outflows and other recycled material but consistent with primordial material that has been mixed with more metal-enriched material. While the uncertainties in our data do not allow us to rule out any of the proposed scenarios they do indicate that the gas being tidal in origin, which seems most likely morphologically, is hard to reconcile with the measurements without at least some significant mixing with low metal- 
licity material. Nevertheless, the data also seem to indicate that pollution of the gas by the galaxies through tidal mixing or outflows is likely, i.e., that the gas is not completely pristine.

This work was supported by the Hubble Grant, HSTGO-12198.01-A. We thank Curtis Struck for his extremely helpful feedback and suggestions for this work. We appreciate Tom Osterloo for his willingness to share the Westerbork maps of the Leo Ring and thank Sabrina Stierwalt and the ALFALFA collaboration (PIs Haynes and Giovanelli) for the Arecibo maps of the Leo Ring and for useful discussions.Brian Kent and John Feldmeir also provided insightful discussions that helped shape this project.

Facilities: HST (COS) 
Table 1

COS Observations

\begin{tabular}{cccr}
\hline \hline Target & $\mathrm{z}$ & Grating & $t_{\text {exp }}(\mathrm{s})$ \\
\hline SDSSJ104816.25+120734.7 & 0.2909 & G130M & 14384 \\
SDSSJ104709.83+130454.6 & 0.3997 & G130M & 8383 \\
SDSSJ104843.49+130605.9 & 0.2185 & G130M & 11435 \\
SDSSJ104843.49+130605.9 & 0.2185 & G160M & 11389 \\
\hline
\end{tabular}

Table 2

SDSSJ 104816.25+120734.7 Absorption Measurements

\begin{tabular}{|c|c|c|c|c|c|c|c|c|c|c|}
\hline Ion & $\begin{array}{r}\lambda \\
{[\AA]}\end{array}$ & $\overline{\mathrm{SL}}$ & $\begin{array}{c}\text { EW } \\
{[\mathrm{m} \AA]}\end{array}$ & $\begin{array}{l}\sigma_{E W} W \\
{[\mathrm{~m} \AA]}\end{array}$ & $\begin{array}{c}\mathrm{b} \\
{[\mathrm{km} / \mathrm{s}]}\end{array}$ & $\begin{array}{c}\sigma_{b} \\
{[\mathrm{~km} / \mathrm{s}]}\end{array}$ & $\begin{array}{c}\log \mathrm{N} \\
{\left[\mathrm{cm}^{-2}\right]}\end{array}$ & $\begin{array}{c}\sigma_{N} \\
{\left[\mathrm{~cm}^{-2}\right]}\end{array}$ & $\begin{array}{c}\mathrm{Vel} \\
{[\mathrm{km} / \mathrm{s}]}\end{array}$ & $\begin{array}{c}\sigma_{V e l} \\
{[\mathrm{~km} / \mathrm{s}]}\end{array}$ \\
\hline N I & 1199.55 & 2.8 & 147 & 116 & 19.9 & 21.7 & 14.3 & 0.4 & 924 & 15 \\
\hline N I & 1200.22 & $\ldots$ & 89 & 119 & $\ldots$ & $\ldots$ & $\ldots$ & $\ldots$ & $\ldots$ & $\ldots$ \\
\hline N I & 1200.71 & $\ldots$ & 59 & 85 & & .. & $\ldots$ & . & & $\ldots$ \\
\hline Si II & 1190.42 & 7.5 & 255 & 153 & 19.1 & 5.4 & 14.4 & 0.5 & 923 & 6 \\
\hline Si II & 1193.29 & $\ldots$ & 445 & 233 & $\ldots$ & $\cdots$ & $\ldots$ & $\ldots$ & $\cdots$ & \\
\hline Si II & 1260.42 & $\ldots$ & 319 & 133 & $\ldots$ & $\ldots$ & $\ldots$ & $\ldots$ & . & $\ldots$ \\
\hline Si II & 1304.37 & $\ldots$ & 175 & 115 & $\ldots$ & $\ldots$ & $\ldots$ & $\ldots$ & $\ldots$ & 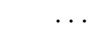 \\
\hline C II & 1334.53 & 2.4 & 485 & 55 & 65.7 & 9.2 & 14.7 & 0.1 & 907 & 7 \\
\hline Si III & 1206.50 & 3.6 & 328 & 194 & 20.0 & 0.0 & 14.3 & 1.8 & 918 & 23 \\
\hline Si IV & 1393.75 & 2.4 & 113 & 136 & 13.7 & 20.8 & 13.6 & 0.9 & 923 & 13 \\
\hline Si IV & 1402.77 & $\ldots$ & 164 & 192 & $\cdots$ & $\cdots$ & $\cdots$ & $\ldots$ & $\cdots$ & $\ldots$ \\
\hline
\end{tabular}

Table 3

SDSSJ 104709.83+130454.6 Absorption Measurements

\begin{tabular}{|c|c|c|c|c|c|c|c|c|c|c|}
\hline Ion & $\begin{array}{r}\lambda \\
{[\AA]}\end{array}$ & $\mathrm{SL}$ & $\begin{array}{c}\mathrm{EW} \\
{[\mathrm{m} \AA]}\end{array}$ & $\begin{array}{l}\sigma_{E W} \\
{[\mathrm{~m} \AA]}\end{array}$ & $\begin{array}{c}\mathrm{b} \\
{[\mathrm{km} / \mathrm{s}]}\end{array}$ & $\begin{array}{c}\sigma_{b} \\
{[\mathrm{~km} / \mathrm{s}]}\end{array}$ & $\begin{array}{c}\log \mathrm{N} \\
{\left[\mathrm{cm}^{-2}\right]}\end{array}$ & $\begin{array}{c}\sigma_{N} \\
{\left[\mathrm{~cm}^{-2}\right]}\end{array}$ & $\begin{array}{c}\mathrm{Vel} \\
{[\mathrm{km} / \mathrm{s}]}\end{array}$ & $\begin{array}{c}\sigma_{V e l} \\
{[\mathrm{~km} / \mathrm{s}]}\end{array}$ \\
\hline Si II & 1190.42 & 9.6 & 206 & 102 & 19.1 & 3.4 & 14.0 & 0.2 & 852 & 4 \\
\hline Si II & 1193.29 & $\ldots$ & 132 & 65 & $\cdots$ & $\ldots$ & $\ldots$ & $\ldots$ & $\ldots$ & $\ldots$ \\
\hline Si II & 1260.42 & $\ldots$ & 236 & 76 & $\ldots$ & $\ldots$ & $\ldots$ & $\ldots$ & $\ldots$ & $\ldots$ \\
\hline Si II & 1304.37 & $\ldots$ & 89 & 42 & $\cdots$ & $\cdots$ & $\ldots$ & $\cdots$ & . & . \\
\hline C II & 1334.53 & 3.4 & 257 & 79 & 47.0 & 14.2 & 14.5 & 0.1 & 864 & 1 \\
\hline Si III & 1206.50 & 2.5 & 257 & 124 & 42.0 & 23.0 & 13.4 & 0.2 & 862 & 1 \\
\hline Si IV & 1393.75 & 2.5 & 110 & 59 & 34.9 & 15.6 & 13.5 & 0.2 & 845 & 1 \\
\hline Si IV & 1402.77 & $\ldots$ & 86 & 55 & 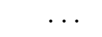 & $\cdots$ & $\cdots$ & $\cdots$ & $\cdots$ & 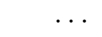 \\
\hline
\end{tabular}

Table 4

SDSSJ 104843.49+130605.9 Absorption Measurements

\begin{tabular}{rrrrrrrrrrr}
\hline \hline Ion & $\begin{array}{r}\lambda \\
{[\AA]}\end{array}$ & SL & $\begin{array}{c}\mathrm{EW} \\
{[\mathrm{m} \AA]}\end{array}$ & $\begin{array}{r}\sigma_{E W} \\
{[\mathrm{~m} \AA]}\end{array}$ & $\begin{array}{c}\mathrm{b} \\
{[\mathrm{km} / \mathrm{s}]}\end{array}$ & $\begin{array}{c}\sigma_{b} \\
{[\mathrm{~km} / \mathrm{s}]}\end{array}$ & $\begin{array}{c}\log \mathrm{N} \\
{\left[\mathrm{cm}^{-2}\right]}\end{array}$ & $\begin{array}{c}\sigma_{N} \\
{\left[\mathrm{~cm}^{-2}\right]}\end{array}$ & $\begin{array}{c}\text { Vel } \\
{[\mathrm{km} / \mathrm{s}]}\end{array}$ & $\begin{array}{c}\sigma_{V e l} \\
{[\mathrm{~km} / \mathrm{s}]}\end{array}$ \\
\hline C II & 1334.53 & 1.5 & 170 & 151 & 64.9 & 46.8 & 14.2 & 0.3 & 842 & 1 \\
Si III & 1206.50 & 2.3 & 312 & 159 & 64.4 & 32.1 & 13.4 & 0.2 & 871 & 23 \\
C IV & 1548.19 & 1.8 & 192 & 157 & 30.7 & 31.9 & 14.0 & 0.4 & 845 & 1 \\
C IV & 1550.78 & $\cdots$ & 170 & 263 & $\cdots$ & $\cdots$ & $\cdots$ & $\cdots$ & $\cdots$ & $\cdots$ \\
\hline
\end{tabular}

Table 5

Metallicity Measurements

\begin{tabular}{cccc}
\hline \hline Sightline & $\begin{array}{c}\text { Atomic } \\
\text { Species }\end{array}$ & $\begin{array}{c}{[\mathrm{X} / \mathrm{HI}]} \\
\text { (uncorrected) }\end{array}$ & $\begin{array}{c}{[\mathrm{X} / \mathrm{H}] /[\mathrm{X} / \mathrm{H}] \odot} \\
\text { (corrected) }\end{array}$ \\
\hline Q104816 & Si II & $-5.1 \pm 0.5$ & $-1.0_{-0.4}^{+0.9}$ \\
Q104816 & C II & $-4.8 \pm 0.1$ & $-1.6_{-0.1}^{+0.5}$ \\
Q104816 & N I & $-5.2 \pm 0.4$ & $-0.8_{-0.6}^{+0.4}$ \\
Q104709 & Si II & $-5.1 \pm 0.2$ & $-1.1_{-0.2}^{+0.7}$ \\
Q104709 & C II & $-4.6 \pm 0.1$ & $-1.0_{-0.1}^{+0.7}$ \\
\hline
\end{tabular}




\section{REFERENCES}

Adams, J. J., Uson, J. M., Hill, G. J., \& MacQueen, P. J. 2011, ApJ 728, 107

Appleton, P. N., Charmandaris, V., \& Struck, C. 1996, ApJ 468 , 532

Asplund, M., Grevesse, N., Sauval, A. J., \& Scott, P. 2009, ARA\&A 47, 481

Bekki, K., Koribalski, B. S., Ryder, S. D., \& Couch, W. J. 2005 MNRAS 357, L21

Bot, C., Helou, G., Latter, W. B.,et al. 2009, AJ 138, 452

Bransford, M. A., Appleton, P. N., Marston, A. P., \& Charmandaris, V. 1998, AJ 116, 2757

Bresolin, F., Kennicutt, R. C., \& Ryan-Weber, E. 2012, ApJ 750, 122

Bresolin, F., Ryan-Weber, E., Kennicutt, R. C., \& Goddard, Q. 2009, ApJ 695, 580

Bruzual, G. \& Charlot, S. 2003, MNRAS 344, 1000

Danforth, C. W., Keeney, B. A., Stocke, J. T., Shull, J. M., \& Yao, Y. 2010, ApJ 720, 976

de Mello, D. F., Urrutia-Viscarra, F., Mendes de Oliveira, C., et al. 2012, MNRAS 426, 2441

Duc, P.-A., Paudel, S., McDermid, et al. 2014, MNRAS 440, 1458

Ferland, G. J., Korista, K. T., Verner, D. A., et al. 1998, PASP 110, 761

Fogarty, L., Thatte, N., Tecza, M., et al. 2011, MNRAS 417, 835

Fox, A. J., Richter, P., Wakker, B. P., et al. 2013, ApJ 772, 110

Gehrels, N.: 1986, ApJ 303, 336

Goddard, Q. E., Bresolin, F., Kennicutt, R. C., Ryan-Weber, E. V., \& Rosales-Ortega, F. F. 2011, MNRAS 412, 1246

Harris, W. E., Harris, G. L. H., Layden, A. C., \& Wehner, E. M. H. 2007, ApJ 666, 903

Haynes, M. P., Giovanelli, R., Martin, A. M., et al. 2011, AJ 142, 170

Higdon, J. L. \& Wallin, J. F.: 1997, ApJ 474, 686

Keeney, B. A., Danforth, C. W., Stocke, J. T., France, K., \& Green, J. C. 2012, PASP 124, 830

Keeney, B. A., Stocke, J. T., Rosenberg, J. L., et al. 2013, ApJ 765, 27
Kriss, G. A. 2011, Improved Medium Resolution Line Spread Functions for COS FUV Spectra, Technical report

Lehner, N., Howk, J. C., Tripp, T. M., et al. 2013, ApJ 770, 138

Madore, B. F., Nelson, E., \& Petrillo, K. 2009, ApJS 181, 572

Mapelli, M. \& Mayer, L. 2012, MNRAS 420, 1158

Michel-Dansac, L., Duc, P.-A., Bournaud, F., et al. 2010, ApJ 717, L143

Morton, D. C. 2003, ApJS 149, 205

Oey, M. S. \& Kennicutt, Jr., R. C. 1993, ApJ 411, 137

Rood, H. J. \& Williams, B. A. 1985, ApJ 288, 535

Sánchez-Blázquez, P., Forbes, D. A., Strader, J., Brodie, J., \& Proctor, R. 2007, MNRAS 377, 759

Schneider, S. 1985, ApJ 288, L33

Schneider, S. E.: 1989, ApJ 343, 94

Schneider, S. E., Helou, G., Salpeter, E. E., \& Terzian, Y. 1983, ApJ 273, L1

Schneider, S. E., Salpeter, E. E., \& Terzian, Y. 1986, AJ 91, 13

Schneider, S. E., Skrutskie, M. F., Hacking, P. B., et al. 1989, AJ 97, 666

Sil'chenko, O. K., Moiseev, A. V., Afanasiev, V. L., Chavushyan, V. H., \& Valdes, J. R. 2003, ApJ 591, 185

Skrutskie, M. F., Shure, M. A., \& Beckwith, S. 1984, ApJ 282, L65

Stanimirovic, S., Staveley-Smith, L., Dickey, J. M., Sault, R. J., \& Snowden, S. L. 1999, MNRAS 302, 417

Stierwalt, S., Haynes, M. P., Giovanelli, R., et al. 2009, AJ 138, 338

Thilker, D. A., Donovan, J., Schiminovich, D., et al. 2009, Nature 457, 990

Torres-Flores, S., de Oliveira, C. M., de Mello, D. F., Scarano, S., \& Urrutia-Viscarra, F. 2012, MNRAS 421, 3612

Tremonti, C. A., Heckman, T. M., Kauffmann, G., et al. 2004, ApJ 613, 898

Werk, J. K., Putman, M. E., Meurer, G. R., \& Santiago-Figueroa, N.: 2011, ApJ 735, 71

Woosley, S. E., Heger, A., \& Weaver, T. A. 2002, RvMP 74, 1015

Wotta, C., Howk, J. C., Lehner, N., \& O'Meara, J. 2014, in $B A A S, 223,243.05$ 